\title{
Les journaux d'Henry Bauchau durant ses années suisses, socles d'une quête identitaire
}

En 1992, Henry Bauchau élargit la palette générique déjà ample de son œuvre en publiant son premier journal, Jour après jour, auquel succéderont cinq volumes qui couvrent le parcours de l'écrivain de 1960 à 2005, exception faite des années 1965 à 1972. Parmi ceux-ci, La Grande Muraille, qui embrasse les années 19601965, et Les Années difficiles, qui s'étend sur la période de 1972 à 1982, constituent des témoignages précieux des années déterminantes qu'Henry Bauchau a passées en Suisse, et plus précisément de celles durant lesquelles il a vécu à Gstaad, village du canton de Berne où se trouvait l'Institut Montesano pour jeunes filles dont il a été le directeur de 1958 à 1975. Déterminantes, ces années en Suisse ne l'ont pourtant pas été en raison de cette fonction qu'il exerça autant par nécessité que par choix, mais parce que c'est là, au cœur des Alpes, loin de sa Belgique natale comme de Paris où il vécut durant six ans après la guerre, que son œuvre a peu à peu vu le jour et qu'avec elle, Henry Bauchau est devenu un écrivain à part entière.

Emblématiques de la singularité de leur auteur et de la spécificité de sa poétique, les journaux d'Henry Bauchau se caractérisent par leur hétérogénéité: accompagnement du roman et de son écriture, lieu même de l'éclosion de la matière romanesque ou poétique, saisie de la fugacité du temps, l'écriture journalière témoigne en outre d'un rapport au monde, et des différentes manières dont le diariste habite ce dernier ${ }^{\mathbf{3 9}}$. Les journaux sont tout cela, et plus encore: notes de lecture ${ }^{\mathbf{4 0}}$, carnets de voyage, témoignages de rencontres avec des anonymes comme avec des figures éminentes de la littérature, ils couvrent tant la vie personnelle de leur auteur, de

\footnotetext{
39 Cf. Myriam Watthee-Delmotte, "De l'aveuglement au sens, Edipe et l'écrivain sur la route. Lecture du journal d'Henry Bauchau", dans Anna Soncini Fratta (a cura di), Henry Bauchau. Un écrivain, une ouvre. Atti del Centro Studi sulla Letteratura Belga di Lingua Francese. Terzo Seminario Internazionale, Noci, Clueb, 1993, "Beløil», pp. 241-254; Raymond Michel, "Le Journal d'Antigone d'Henry Bauchau ou les mouvements de l'écriture», dans Pierre Halen, Raymond Michel et Monique Michel (dir.), Henry Bauchau, une poétique de l'espérance. Actes du colloque international de Metz (6-8 novembre 2002), Berne, Peter Lang, 2004, Coll. "Recherches en littérature et spiritualité», pp. 179-218; Jean Leclercq, "Du poème qui n'est pas de ce monde», dans Catherine Mayaux et Myriam Watthee-Delmotte (dir.), Henry Bauchau, écrire pour habiter le monde, Saint-Denis, Presses universitaires de Vincennes, 2009, Coll. "L'Imaginaire du Texte», pp. 27-38.

40 Cécilia Dejean, Jour après jour d'Henry Bauchau: Journal d'un écrivain-lecteur, Mémoire de master en littérature du monde francophone, Université de Cergy-Pontoise, juin 2007.
} 
ses rêves ${ }^{\mathbf{4 1}}$ à une réalité souvent perçue douloureusement, que les événements de la vie politique et de l'histoire mondiale.

La plupart des études menées sur les journaux de l'écrivain belge ont mis en avant les liens de ces derniers à l'œuvre et à l'écriture, dans la mesure où ces carnets de notes atypiques constituent un témoignage rare sur le processus de création des poèmes et des romans. Ces approches font ainsi écho à la volonté d'Henry Bauchau de mettre en avant l'œuvre plutôt que lui-même, et ce jusque dans ses journaux dont certains titres ou sous-titres sont à cet égard éloquents: le Journal d'Antigone ou La Grande Muraille. Journal de La Déchirure, mettent d'abord l'accent sur l'œuvre publiée dont ils restituent les années d'écriture, tout comme Jour après jour, volume inaugural des journaux, l'avait fait pour Edipe sur la route. C'est que l'écrivain, qui se montre souvent réticent à une lecture trop biographique de l'œuvre, semble préférer braquer le projecteur sur la création et son objet plutôt que sur le sujet créateur et l'aspect factuel de son parcours, ainsi qu'il le pointe dans la préface de La Grande Muraille:

Les années soixante sont maintenant lointaines, ce journal ne tente pas de les dire. Les événements extérieurs et ceux de ma propre vie y tiennent peu de place. Ces pages ont été écrites pour accompagner la difficile entreprise et le parcours sinueux d'un premier roman. ${ }^{42}$

Si, dans le travail a posteriori de publication des journaux, le diariste, de son propre aveu, retravaille très peu son style, il coupe, il réduit ${ }^{43}$, et l'on peut supposer qu'il supprime essentiellement ce qui le concerne trop directement pour insister sur le processus de création de l'œuvre. Il suit en cela l'exemple des journaux de Jünger dont lui a parlé le critique Jean-Pierre Jossua, lors d'un séjour chez les Stein en Bretagne, et qui suscitent d'emblée son adhésion:

Jean-Pierre Jossua parle de la forme du Journal dans lequel il distingue le Journal intime, le Journal d'idées et le Journal littéraire dont il trouve que l'exemple le plus réussi est celui de Jünger. La définition me semble juste, Jünger ne nous livre pas ses confidences mais des sentiments, des pensées, des choses vues choisis par lui et auxquels il a donné forme pour la publication [...]. Mes notes, si un jour je parviens à les reprendre, ne devraient pas être publiées intégralement. Il y a beaucoup de déchets, je devrais y faire un choix, les polir, c'est l'exemple de Jünger que je devrais suivre. ${ }^{44}$

41 Geneviève Henrot-Sostero, "La voix de l'ombre. Pouvoirs du rêve dans les journaux d'Henry Bauchau", dans $N u(e), n^{\circ} 35$ (numéro coordonné par Myriam Watthee-Delmotte), mars 2007, pp. 247-260.

42 Henry Bauchau, La Grande Muraille. Journal de La Déchirure (1960-1965), Arles, Actes Sud, 2005, Coll. "Babel», p. 11. Toutes les citations ultérieures sont issues de cette édition, sauf mention contraire. 43 « L'écriture de mon journal est très différente de celle de mes romans ou de mes poèmes. Pour le journal, je coupe, je change parfois des phrases défectueuses, mais je ne réécris pas; alors que pour mes romans, je réécris énormément, je fais toujours plusieurs versions. Dans le journal, je note des choses qui me viennent comme ça, sans plan. J'écris.", Henry Bauchau, "Entretien avec G. Moreau ", dans Les Moments littéraires. Dossier Henry Bauchau, n 14, juillet 2005, p. 22.

44 Henry Bauchau, Les Années difficiles. Journal 1972-1983, Arles, Actes Sud, 2009, pp. $250-251$. 
Parallèlement à l'acte d'écriture et à la genèse de l'œuvre que les journaux bauchaliens placent au premier plan, se joue néanmoins la naissance d'un sujet écrivant, d'autant plus perceptiblement dans les journaux suisses d'Henry Bauchau qu'ils couvrent la période de ses premiers pas sur la scène littéraire. La préface de La Grande Muraille l'énonce d'ailleurs en toutes lettres:

Avant [La Déchirure], j'étais déjà un écrivant, je n'étais pas encore un écrivain. Je n’avais pas confié à l'écriture l'opacité, la ténacité, l'espérance de ma vie. Ce journal dit un peu comment je suis devenu celui que je n'étais pas encore. ${ }^{45}$

Durant ces années incertaines et déterminantes que l'écrivain naissant passe en Suisse, c'est un peu comme si le journal, en éclairant Henry Bauchau "sur ce qui [lui] est advenu ou qui [lui] adviendra encore " ${ }^{\mathbf{4 6}}$, remplissait un rôle de garde-fou: face à l'indétermination de l'écriture, ces carnets de notes quotidiennes apparaissent comme l'occasion pour le diariste de se donner peu à peu une cohérence identitaire, passant du statut d'“écrivant" à celui d'“écrivain", transformant son activité en identité. Ce qui s'est joué en Suisse, loin des milieux littéraires de la vie parisienne, au cours de cette "seconde vie "47 que Bauchau a voulu se donner, a trait à une quête identitaire, à laquelle le journal a largement contribué en devenant le lieu privilégié de représentation de soi en tant qu'écrivain. C'est à ces représentations, à ces images de l'écrivain et de ses pratiques, telles qu'elles se déploient largement dans les journaux, que cette étude va s'attacher.

\section{La Grande Muraille : de l'écrivant à l'écrivain}

Si, dans La Grande Muraille, le journal concentre les efforts du sujet vers une certaine forme de cohérence identitaire, c'est qu'il vise précisément à compenser un doute, un manque, un éclatement de soi que contribue à entretenir la "perpétuelle tendance à la dépression " ${ }^{48}$ d'Henry Bauchau. "[E]n écrivant», dit-il, "ce que je tente encore c'est de me réaliser " ${ }^{\mathbf{4 9}}$, phrase programmatique qui laisse entendre à la fois le désir d'épanouissement de l'écrivain et sa volonté de s'ancrer dans le réel, de se rendre davantage réel. Écho des schémas initiatiques qui organisent l'univers romanesque bauchalien, entendus comme succession de morts symboliques et de renaissances, le journal pose la nécessité d'organiser sa vie entre un avant - un sujet écrivant - et un après - un sujet écrivain. Le diariste y chante le tenace espoir

Toutes les citations ultérieures sont issues de cette édition, sauf mention contraire. 45 La Grande Muraille, p. 11.

46 "Entretien avec Henry Bauchau ", dans L'Eil-de-Bœuf, n 17, avril 1999, p. 7.

47 Cf. Les Années difficiles, p. 37: "mes premières années à Gstaad. Alors, à trente-sept ans je croyais avoir raté la première partie de ma vie et j'espérais me reprendre dans la seconde».

48 La Grande Muraille, p. 37.

49 Ibid., p. 68. 
de changement qui le soutient et qui trouve sa source dans la confiance qu'il a de porter une ouvre forte, celle qui, dit-il, "donne tout son sens à [s]a vie „\$0

Pourtant, l'identité d'écrivain, par son absence de bornes, "est sans doute ce qui peut s'approcher le plus d'un état d'indétermination "51, comme le relève Nathalie Heinich au cours de son étude sur la représentation identitaire des écrivains. Henry Bauchau, au fil des pages de son journal, dans la solitude et la discipline qu'il s'impose, en fait régulièrement le constat:

Je sais bien que la voie de la création est moins parfaite et sereine que d'autres. Elle est faite de déchirements, de harassements, de bonds dans la lumière, suivis d'épaisses ténèbres. C'est la mienne pourtant et je n'en ai pas d'autre. ${ }^{\mathbf{5 2}}$

Entre se dire et se vivre écrivain, l'écart est grand: derrière la cohérence identitaire d'une catégorie sociale, quête d'un illusoire idéal, se cachent nombre de fractures que La Grande Muraille met en lumière à de nombreuses reprises.

Un facteur, et non des moindres, qui contribue à ce sentiment de flottement identitaire réside dans le fait qu'Henry Bauchau, en 1960, n'a pas encore pu s'inscrire comme il le voulait dans le champ littéraire: sa bibliographie ne compte qu'une publication, Géologie, qu'il a cependant éditée dans la prestigieuse collection NRF de Gallimard et qui lui a valu le prix Max Jacob. S'y adjoint bientôt sa pièce de théâtre, Gengis Khan, écrite en 1956 et publiée chez Mermod en 1960. Mais si ces livres et la matérialité qu'ils donnent à l'œuvre contrecarrent le manque de consistance de l'identité de l'écrivain, ils ne lui octroient nullement l'assurance dans sa démarche, d'autant qu'ils sont tombés dans un relatif silence, sans tirer leur auteur de l'obscurité pour lui donner le succès escompté53. Afin de compenser ce déficit de confiance, Henry Bauchau se livre, consciemment ou non, à des stratégies consignées dans le journal et destinées à lui octroyer, peu à peu, la cohésion d'une identité d'écrivain, fût-elle illusoire.

L'une des plus répétées se joue dans les lectures et les prises de notes régulières qu'en fait Henry Bauchau. Si son goût le tourne vers les grands canons de la littérature ${ }^{54}$, tels Hölderlin, Nietzsche, Baudelaire ou Rimbaud, ses réactions, entre admiration et comparaison, témoignent d'un sentiment fort d'appartenance à la

\footnotetext{
50 Ibid., p. 296.

51 Nathalie Heinich, Être écrivain. Création et identité, Paris, La Découverte, 2000, Coll. "Armillaire", p. 92.

52 La Grande Muraille, p. 255.

53 " Tout cela [les représentations de Gengis Khan à Paris en 1961] m'a fait mesurer les extraordinaires illusions qui ont été les miennes en 1955 quand j'ai cru que cette pièce me tirerait de l'obscurité, me donnerait le succès et peut-être une autre vie. En réalité c'est une pièce de ma jeunesse d'écrivain, où j'ai fourré tous les problèmes de mes rapports avec l'inconscient. Il se peut qu'elle soit reprise un jour mais il est dorénavant bien clair que ce n'est pas elle qui me donnera le succès ni la possibilité de me consacrer surtout à mon travail d'écrivain", ibid., pp. 130-131.

54 Ainsi que le relève Cécilia Dejean dans Jour après jour d'Henry Bauchau: Journal d'un écrivainlecteur, op. cit., p. 60.
} 
même communauté que ces derniers, manière de se construire comme écrivain par stratégie d'assimilation:

En le lisant j'ai ressenti une fraternité profonde avec Hölderlin. Certes je n'ai pas son attitude aérienne, ni sa profondeur soudaine et pourtant j'appartiens au même monde que lui. 55

Ou encore, à propos du Journal de Delacroix, l'attitude de l'écrivain oscille cette fois entre distance admirative et sentiment d'identification: "J'avance le soir dans le Journal de Delacroix. Que le contact direct avec les esprits supérieurs est fécondant "56, écrit-il le 9 octobre 1961, avant d'ajouter quelques jours plus tard: "En lisant ce Journal ce n'est pas seulement Delacroix que j'apprends à connaître, c'est moimême. " ${ }^{57}$ Dans la majorité des notes de lecture relevées dans le journal, ou bien l'écrivain, porté à l'assimilation, se conforte dans ses choix esthétiques et ses intuitions artistiques en trouvant des échos de ceux-ci chez des écrivains confirmés, ou bien, selon le schème inversé de la différenciation, il précise les lignes de sa poétique par opposition à celle d'un auteur encensé: "Je relis Rimbaud, l'extraordinaire assurance de sa démarche. Il va, il n'y a pas de "mais". L'autre point de vue m'est toujours présent et le "mais" m'empêtre. „58 Dans cette relation contradictoire à la tradition, Henry Bauchau acquiert peu à peu la conscience d'appartenir à une communauté, ce qui ne va pas sans jouer un rôle de cohésion dans sa construction identitaire.

Celle-ci est pourtant massivement mise à mal par la position dans laquelle Henry Bauchau se trouve pour exercer son activité d'écrivain. Pour assurer moins sa propre indépendance financière que celle de sa famille, il est en effet contraint d'exercer parallèlement à l'écriture la profession de directeur d'un institut pour jeunes filles à Gstaad. L'écriture découle alors d'un compromis sur le temps, étant donné que Bauchau doit se résigner à voir diminuer les plages horaires imparties à celle-ci au profit d'une autre activité. Si son métier lui donne l'avantage d'une certaine indépendance, puisque, grâce à ses revenus fixes, il peut étaler la rédaction de ses livres sur une période aussi ample qu'il le désire, ou opter pour des sujets ou des genres littéraires sans être tenu par une obligation de succès ${ }^{59}$, il n'en est pas moins vécu dans la souffrance, dans l'obligation subie d'un dédoublement d'où émerge le régulier ton de plainte de La Grande Muraille ${ }^{\mathbf{6 0}}$ :

55 La Grande Muraille, p. 158.

56 Ibid., p. 159.

57 Ibid., p. 163.

58 Ibid., p. 295.

59 Ibid., pp. 277-278: "Très énervé - et stupidement - hier et aujourd'hui par le sentiment que je n'ai presque pas le temps de travailler. [...] Mais quoi, je n'écrirai pas de nombreux ouvrages [...]! Quelle importance si j'arrive à en faire un ou deux de bons!»

60 À propos du ton de plainte qui émane des journaux d'Henry Bauchau, lire: Déborah Gabriel, «Les journaux, l'autre voix de l'écriture", dans Myriam Watthee-Delmotte et Jacques Poirier (dir.), Pierre Jean Jouve et Henry Bauchau: les voix de l'altérité, Dijon, Presses universitaires de Dijon, 2006, Coll. "Écritures", pp. 199-212. 
Étrange que mes actes extérieurs pour le moment soient si cohérents: vraiment ceux d'un directeur d'école et qu'en même temps je sois la proie du plus complet désordre intérieur. ${ }^{61}$

Alors que son rôle de directeur à Montesano, qu'il considère comme secondaire par rapport à ses aspirations d'écrivain, se décline soit sur un ton de désespoir, soit sur celui d'un plaisir coupable car, lui semble-t-il, coupé de l'essentiel ${ }^{\mathbf{6 2}}$, le journal tâche de se concentrer sur l'écriture, qui seule mérite d'être désignée par le terme de "travail", entendu tantôt dans son sens noble de labeur artisanal, tantôt dans son acception sacrificielle, à la mesure de l'effort investi. Au fil des pages de La Grande Muraille, l'insistance sur le "travail», vecteur de cohésion identitaire - "c'est dans le travail [...] que je trouve équilibre et réponse» ${ }^{\mathbf{6}}$, note Henry Bauchau, ou encore: "Dans la masse des incertitudes qui m'entourent me cramponner à cette certitude: mon travail "64 l'écrivain dans sa posture professionnelle: celle-ci, dans ce cas précis, se traduit notamment par l'importance accordée à la capitalisation des pages écrites quotidiennement et par le sentiment de culpabilité qui pointe chez le scripteur dès que son objectif journalier n'est pas atteint ${ }^{\mathbf{6 5}}$. La valorisation de cette posture professionnelle, où le volontarisme dans le travail permet de pallier les doutes sur soi en tant que créateur, éclipse dès lors quelque peu dans le journal la posture inspirée, que Nathalie Heinich situe à l'autre bout de l'échelle des valeurs. Ces deux postures ne sont pourtant pas inconciliables, loin s'en faut, et Pierre Jean Jouve, l'un des modèles de Bauchau, montre d'ailleurs dans son journal En miroir combien, tout en étant un écrivain inspiré, il se doit de consacrer des heures à son travail pour parvenir au résultat désiré. Le "monde inspiré» $\mathbf{6 6}^{\mathbf{6}}$ est pareillement primordial dans l'axiologie d'Henry Bauchau ${ }^{\mathbf{6 7}}$, qui semble surtout satisfait lorsque la création se fait hors de toute intervention de sa part. Mais ce monde apparaît moins couramment dans ce premier journal, voué à la construction d'une cohésion identitaire. Peutêtre la cause en est-elle que, contrairement au travail, l'inspiration n'offre aucune prise de contrôle, et plonge le sujet créateur dans des abîmes d'incertitude au lieu de le structurer:

61 La Grande Muraille, pp. 245-246.

$62 \mathrm{Ibid}$., p. 339: "Belle inconséquence. Pour compléter le tableau je pallie ma faiblesse et mon indécision sur le terrain en me montrant homme d'action, précis, travailleur sur le terrain secondaire, celui de l'école."

63 Ibid., p. 77.

64 Ibid., p. 375.

65 Ibid., p. 51 : «Je me sens un peu coupable quand je n'arrive pas, au bout de la journée, à produire une certaine quantité de petits signes noirs sur une page blanche."

66 Cf. Luc Boltanski, Laurent Thevenot, De la justification: les économies de la grandeur, Paris, Gallimard, 1991.

67 Il reconnaîtra d'ailleurs dans les pages ultérieures des Années difficiles se sentir proche de l'idéal romantique, qui a répandu l'image stéréotypée de l'écrivain inspiré: "Ce romantisme, c'est bien ce qui bouillonne au fond de moi-même", Les Années difficiles, p. 31. 
Quel long chemin pour retrouver l'inspiration. Je brode, je tricote, j'attends les mots et le rythme pour les porter. Je connais bien pourtant cet état de vide dont on ne sort qu'en empoignant soudain le travail comme on prend un outil. ${ }^{68}$

Enfin, si c'est la nécessité d'exercer un métier qui a amené Henry Bauchau à s'installer en Suisse, cette situation géographique ne résout en rien la difficulté de l'écrivain à se définir et à se vivre comme tel. Venu du Paris des années d'aprèsguerre où il a eu l'occasion, par son activité dans le monde de l'édition, de pénétrer dans certains cercles littéraires, il se retrouve à Gstaad qui, hors des réseaux traditionnels de la culture, souffre de la comparaison. C'est pourtant dans ce lieu reculé et, dès lors, selon le schème de l'exclusion, que Bauchau a aménagé sa vie d'écrivain. Dans son écrin de montagnes, Gstaad a notamment été le lieu où s'est ravivé le grand dialogue de l'écrivain avec la nature, écho inattendu de la nature brabançonne de son enfance ou de celle, plus ancestrale encore, de ce lieu mythique qu'il nomme «la Chine intérieure». Néanmoins, Gstaad apparaît surtout aux yeux de l'écrivain comme le lieu de sa solitude, terme qui revient à plusieurs reprises sous sa plume. Cette solitude, topos de la représentation identitaire de l'écrivain qu'a notamment mis en lumière l'enquête de Nathalie Heinich, est intensément et douloureusement vécue par Henry Bauchau qui, tout au long de son journal, insiste à plusieurs reprises sur sa sensibilité au travail d'équipe ${ }^{69}$ et sur sa revendication à l'intérêt des autres pour son activité d'écrivain.

Le journal dépeint notamment deux stratégies pour aller contre ce sentiment d'isolement et son corollaire, la perte de confiance en son statut d'écrivain. D'une part, Henry Bauchau tente de maintenir et d'élargir un certain réseau de personnalités littéraires et artistiques autour de lui, parmi lesquelles se trouvent au premier plan Pierre Jean Jouve et Jean Amrouche. Il croise également à l'époque la route de l'éditeur Henry-Louis Mermod, de Francis Ponge, de Pierre Skira ou encore de Theodor Adorno, pour ne citer qu'eux. En outre, il n'hésite pas à pallier le manque de résonance rencontré dans son travail en sollicitant toute une série de proches à devenir lecteurs de son ouvre en chantier. C'est ainsi que sont consignées dans le journal nombre de scènes de lecture, très régulièrement faites à voix haute par Bauchau lui-même, saluées généralement par les encouragements de ce public restreint, tantôt constitué de membres de la famille, tantôt de fins connaisseurs, tel Jean Amrouche, que Bauchau qualifie de «vrai lecteur» ${ }^{\mathbf{7 0}}$ :

68 La Grande Muraille, p. 248.

69 Ibid., p. 244: "À cet égard tout est bien changé depuis quelques années. L'espoir de m’insérer dans une équipe ou un effort collectif est mort. Plus personne ne s'intéresse à ce que je fais au moment où je puis vraiment faire des œuvres valables. Cette nudité n'est peut-être pas un mal, mais c'est une souffrance, la brisure d'un perpétuel désir d'échange et de confiance.» Ce sentiment de solitude, exacerbé dans le journal, doit pourtant être relativisé : Henry Bauchau, dont le goût des actions collectives s'est illustré durant ses activités dans le monde chrétien de l'entre-deux-guerres et dans le Service des Volontaires du Travail pour la Wallonie, a également tenté d'insuffler un tel esprit de groupe dans les projets (professionnels ou non) qu'il a menés à Gstaad, comme en ont témoigné Jacques Devriend, le Dr Dreyfuss et d'anciennes élèves de Montesano.

70 Ibid., p. 252. 
[...] j’ai lu à Jean Amrouche le début du roman. Il m’a dit: "C'est très beau [...] Le ton est très simple et juste.» [...] J'avais une certaine appréhension à lui montrer ce que j'avais fait car je sentais qu'il n'aimait qu'à moitié ce que j'ai écrit jusqu'ici. Comme je le sais incapable de cacher ses sentiments j'ai été très encouragé par sa réaction, au moment où je traverse une période de doute pénible. ${ }^{11}$

D'autre part, outre ces scènes de lecture d'où émane le sentiment d'intérêt des autres pour son travail, Henry Bauchau, face à la solitude qui l'accable, prend à quelques reprises le parti d'inverser cette dernière en positivité, pressentant qu'elle est la garantie d'une autodiscipline indispensable pour mener à bien son projet romanesque:

Depuis que j'ai repris le roman et que je me traque constamment pour ne pas perdre de temps je suis curieusement seul. J'ai réduit mes contacts professionnels à l'indispensable et je n'ai plus le temps de parler longuement à personne. Cette solitude n'est pas vide mais bruissante de messages du vécu. ${ }^{72}$

Ainsi, refuge d'un écrivain en mal d'identité et de reconnaissance, du fait de sa solitude, de son manque d'expérience, de l'exercice de son métier, le journal La Grande Muraille s'affirme comme le lieu privilégié pour pallier ces déficiences, mettant en exergue diverses stratégies qui permettent à Henry Bauchau de s'appréhender chaque jour davantage comme un véritable écrivain.

\section{Les Années difficiles: de la désillusion à la révolution intérieure}

Les Années difficiles, second journal d'Henry Bauchau du point de vue de la chronologie biographique, se présente sous une forme légèrement différente de celle des volumes auxquels il succède du point de vue de la publication, n'étant paru qu'en 2009. Non précédé d'une préface, qui dans La Grande Muraille mettait en avant le caractère construit, retravaillé du journal, il donne l'impression de plus se présenter dans un état brut, comme si l'auteur avait pratiqué moins de coupes dans son travail d'édition, assumant peut-être mieux l'intérêt porté à sa biographie à la suite de la consécration du Boulevard périphérique (2008) et de la reconnaissance toujours plus large de son ouvre. Peut-être aussi Henry Bauchau a-t-il moins retravaillé les manuscrits de ses journaux du fait de son âge et de la diminution de ses forces. Le diariste, d'ailleurs, abandonne pour ce Journal, comme c'était déjà le cas pour Passage de la Bonne-Graine et Le Présent d'incertitude, le découpage périodique calqué sur le temps de rédaction d'une ouvre de fiction, comme s'il faisait moins qu'avant de l'œuvre le centre unique de son écriture journalière.

Commençant six ans après la parution de La Déchirure, Les Années difficiles

71 Ibid., pp. $82-83$.

72 Ibid., p. 310. 
dressent un tableau quelque peu différent de l'écrivain et de ses pratiques, où se manifestent notamment certains signes de confiance d'Henry Bauchau en son œuvre et certaines marques de crédit venues des autres: Flammarion lui passe notamment commande d'une biographie, qui deviendra l'Essai sur la vie de Mao Zedong, Gallimard lui fait «[l]e premier versement de quelque importance reçu pour un de [s]es livres» ${ }^{73}$ et lui annonce la première traduction d'une de ses ouvres, Le Régiment noir, en langue espagnole; la Belgique lui décerne le prix FranzHellens. Pourtant, après plus de vingt années consacrées à l'écriture, le bilan se révèle toujours aussi peu satisfaisant pour l'écrivain, qui, alors qu'il croyait pouvoir toucher plus largement le public par ses romans, vit très mal le «mur de silence» ${ }^{74}$ de la critique autour de ses parutions, d'autant qu'il considère à chaque nouvelle publication avoir écrit "sa meilleure ouvre»75. Ainsi, si d'un côté la production de l'écrivain ne cesse de s'accroître, elle n'en provoque pas pour autant, de l'autre, un surcroit de renommée, ce qui plonge Henry Bauchau dans un profond désarroi, lui qui confesse régulièrement sa sensibilité au renom ${ }^{76}$. Le journal, ici encore, n'est pas seulement le lieu de ce constat, mais également celui où l'écrivain concentre ses efforts pour reprendre pied et se reconstruire.

Les modalités de cette reconstruction diffèrent néanmoins de celles mises en œuvre dans La Grande Muraille, et s'inscrivent dans le contexte personnel plus large d'un désastre financier qui mène lentement Henry Bauchau à la faillite. À cela s'ajoutent le fait que l'abandon de son métier à la suite de la fermeture de l'école en 1972 n'apporte pas à Henry Bauchau la libération tant espérée de son travail d'écriture, et le fait que, parallèlement, se renforce le sentiment d'être en marge des milieux littéraires et artistiques: le temps du foisonnement des visites prestigieuses semble loin, et la solitude, dans l'isolement de Gstaad, n'en devient que plus accablante. Afin d'aller vers l'acceptation de cette nouvelle donne, l'écrivain se tourne alors vers une voie de renoncement, de pauvreté et de création "pure»" posture de dévouement à la création où il s'efforce de se détourner de toute compromission avec les contingences mondaines: "L'exemple de Proust et de sa réclusion volontaire doit me guider. Puisque rien d'autre ne s'ouvre il faut que je poursuive mon œuvre, c'est cela qui m'est demandé. „ ${ }^{\mathbf{7 8}}$

Alors que La grande muraille montrait l'importance de la pensée volontariste du philosophe Alain dans la pratique de l'écriture, Les années difficiles mettent à

73 Les Années difficiles, p. 47.

74 Ibid., p. 33.

75 Ibid., p. 150: "j'étais persuadé que c'était [La Chine intérieure] ma meilleure œuvre (mais je l'ai cru de chacun de mes livres), j'éprouve maintenant un dégoût pour elle. Je n'ai même pas envie de l'ouvrir pour me refaire une opinion."

76 Ibid., p. 86: "Le soir coup de téléphone d'un poète canadien qui m'invite à un congrès d'écrivains à Montréal. Cela m'a fait plaisir. Comme une confirmation de mon petit renom d'écrivain. Que je suis sensible à cela."

77 Cf. Nathalie Heinich, op. cit., p. 41.

78 Les Années difficiles, p. 195. 
l'honneur une autre figure non littéraire, Simone Weil, et sa pensée du renoncement en laquelle Henry Bauchau trouve un écho à ses propres aspirations: "Chez Simone Weil il me semble voir une autre voie qui est celle de l'acceptation de tout ce qui arrive y compris la douleur et la destruction. "79 Cette voie d'acceptation constitue l'un des signes de la "révolution intérieure ${ }^{\mathbf{8 0}}$ que l'écrivain est en train d'opérer et de la quête spirituelle qu'il poursuit durant ces "années difficiles" où se ralentit parallèlement, et en partie volontairement, sa production littéraire ${ }^{\mathbf{8 1}}$ : "Quant à l'écriture et à la peinture," note-t-il d'ailleurs, "il faut que je me persuade que cela peut attendre, ce qui se passe en moi est plus important. „82 Outre la lecture de Simone Weil, il creuse dans d'autres directions, notamment grâce à son essai sur Mao qui lui permet d'effectuer un important travail documentaire sur l'Extrême-Orient, ou encore par la reprise de la lecture de la Bible et la redécouverte de bribes de sa foi chrétienne:

Quelque chose a cédé en moi, je ne sais pas plus qu'avant ce qu'est Dieu, toutes les constructions de la foi me passent pour le moment au-dessus de la tête et pourtant je sens non que je m'engage mais que je suis engagé dans un processus et que je m'en trouve bien. ${ }^{\mathbf{3}}$

Portée par le désir de se libérer de ses "revendications intérieures» ${ }^{\mathbf{8 4}}$, de "[s]a mégalomanie et de la tristesse provoquée par le contraste entre ce [qu'il a] rêvé et ce qu'est [s]a vie réelle ${ }^{\mathbf{8 5}}$, cette quête spirituelle va résolument dans le sens d'un "laisser-faire", d'un relâchement, d'une acceptation de "ce qui a été» ${ }^{\mathbf{8 6}}$. Perceptible dans les lectures évoquées par l'auteur, elle se trouve surtout portée par les deux grands ouvrages qui marquent la fin de la période suisse d'Henry Bauchau: La Chine intérieure et l'Essai sur la vie de Mao Zedong. Emblématiques l'un et l'autre de l'espoir d'une révolution personnelle, ils se présentent à plusieurs reprises sous la plume de l'écrivain comme des "épreuves» nécessaires vers la transformation intérieure. "Le poème m'apparaît de plus en plus comme une grande entreprise par laquelle il faut passer coûte que coûte avant de changer de vie et de commencer une autre ouvre» ${ }^{\mathbf{8 7}}$, écrit Bauchau à propos de La Chine intérieure, plusieurs fois comparée à Géologie en ce qu'elle suscite le même espoir d'un nouveau départ. De même, à propos de l'essai sur Mao, l'auteur note: "C'est à nouveau un barrage qu'il

\footnotetext{
79 Ibid., p. 128.

80 Ibid., p. 95.

81 Ibid., p. 137: "Après la tension causée en moi par la mauvaise qualité de ce que j'écrivais et par ma stérilité depuis plus de trois mois, ce refus avait plutôt un caractère apaisant. Depuis je suis triste mais plus serein."

82 Ibid., p. 14.

83 Ibid., p. 132.

84 Ibid., p. 21.

85 Ibid., p. 66.

86 Ibid., p. 226: "J'ai tant désiré la gloire, je la désire peut-être encore et pourtant elle m'eût sûrement fait dérailler si elle était venue du temps de Gengis Khan. C'est ici qu'il faut comprendre cette pensée si difficile de Simone Weil qu' "il faut aimer ce qui a été", oui, y compris toutes mes erreurs." 87 Ibid., pp. 71-72.
} 
faut franchir pour que la voie s'ouvre à de nouvelles ouvres et à de nouvelles perspectives. \$8 ${ }^{\mathbf{8 8}}$ Finalement, à l'image de ce qui a été amorcé dans La Grande Muraille mais selon des modalités qui en diffèrent légèrement, notamment pour ce qui a trait au renoncement et au laisser-faire, Les Années difficiles dressent le tableau d'une vie à renégocier, à redéfinir sans cesse, faite d'épreuves où transparaissent les nécessaires étapes d'une forme d'initiation et de son lot de morts symboliques et de renaissances successives. Dans ce cadre, l'identité d'écrivain, tel un impossible point d'arrivée, ne semble jamais pouvoir être atteinte avec certitude: «il me semble être toujours en train de commencer ma vie» ${ }^{\mathbf{8 9}}$, énonce d'ailleurs Bauchau, à l'âge de soixante-quatre ans.

\section{Une identité en chemin}

L'étude que nous avons amorcée sur les représentations de l'écrivain, de son identité et de ses pratiques telles qu'elles s'esquissent dans le journal, trouverait un juste prolongement dans la confrontation de ce discours diaristique avec les romans d'Henry Bauchau où évoluent des figures d'écrivain, et particulièrement La Déchirure et Le Boulevard périphérique, dont la période de rédaction coüncide avec celle de ces deux journaux ${ }^{\mathbf{9 0}}$. À cet égard, un trait commun unit ces deux romans: ils mettent l'un comme l'autre en avant des figures prétendant ne pas écrire le livre que le lecteur a sous les yeux, comme si l'élaboration de celui-ci se faisait en dehors de ces écrivains-narrateurs, hors de leur maîtrise, émanant d'une instance extérieure indéterminée. Ainsi le narrateur de La Déchirure, jamais perçu en posture de rédaction, évoque bien plutôt "son livre abandonné chez lui»"1, alors que le narrateur du Boulevard périphérique énonce à plusieurs reprises dans le récit son refus ou son incapacité de prendre la plume, instaurant un jeu complexe dans le dispositif narratif du roman par l'inadéquation de l'énonciation et de l'écriture, perceptible notamment dans la citation suivante: "Je n'écrirai pas aujourd'hui, non je n'écrirai pas „\$2. La parole hors-cadre qui se déploie dans ces romans met ainsi en avant moins un sujet créateur qu'une écriture venue d'une source inconnue, reprise du motif tant valorisé par Henry Bauchau d'une création qui se fait en dehors de soi et où le sujet écrivant ne vient qu'au second rang.

La valorisation de la création, du processus de création en tant que tel, qui transparaît en définitive dans de nombreux écrits d'Henry Bauchau, tant fictionnels que référentiels, s'inscrit dans un contexte littéraire spécifique: si le XVIII ${ }^{\mathrm{e}}$ siècle

88 Ibid., p. 191.

89 Ibid., p. 291.

90 Rappelons, en effet, que Le Boulevard périphérique, paru en 2008, est écrit dans une première version au début des années 1980, alors qu'Henry Bauchau a quitté depuis cinq ans la Suisse pour Paris. 91 Henry Bauchau, La Déchirure, Arles/Bruxelles Actes Sud/Labor, Coll. "Babel»/« Espace nord" (rééd.), 1986, p. 94.

92 Henry Bauchau, Le Boulevard périphérique, Arles, Actes Sud, 2008, p. 62. 
s'est notamment conclu par la mise en avant moderne de la figure du créateur, ainsi que l'a mis en lumière Jean-Claude Bonnet ${ }^{\mathbf{3}}$, et alors que cette valorisation a trouvé un prolongement indéniable au XIX siècle qu'a notamment éclairé Paul Bénichou dans son ouvrage intitulé Le sacre de l'écrivain ${ }^{\mathbf{4}}$, le $\mathrm{XX}^{\mathrm{e}}$ siècle a quant à lui débouché sur la valorisation de la création en tant que telle. Comme le note Nathalie Heinich:

On ne cesse ainsi de remonter vers l'amont: de l'œuvre créée à son créateur, et de son créateur à la création même, dont il n'est plus [...] que le médiateur plus ou moins inspiré. 95

Dans cette lignée, les journaux d'Henry Bauchau que nous avons analysés se présentent d'abord comme des récits de création: "récits", d'une part, parce qu'ils tentent d'instaurer, selon le critère de Paul Ricour, une forme de cohérence, notamment identitaire, dans la discordance, et "de création", d'autre part, en ce qu'ils s'attellent à suivre l'élaboration des ouvres depuis le début jusqu'à l'étape finale de la publication. Introspections méticuleuses de l'expérience de l'écriture, les témoignages que nous livre Bauchau dans ses journaux présentent celle-ci comme une forme d'initiation sans cesse répétée. À travers elle, le sujet créateur est amené à opérer une "véritable mutation ontologique", passant, dans La Grande Muraille, du statut d'écrivant à une identité d'écrivain et, dans Les Années difficiles, du sentiment d'échec à la nécessité de s'engager sur la voie d'une révolution intérieure. Si le journal permet de suivre la genèse de l'œuvre, une autre genèse est donc en cours qui redonne une place au sujet créateur: celle de la progressive et toujours inchoative naissance de l'écrivain à lui-même. Dans cet effort soutenu d'individuation du sujet se met au jour, ainsi que nous avons essayé de le montrer, une identité d'écrivain, identité résolument évolutive, toujours en chemin, écho du questionnement identitaire dans lequel sont engagés la plupart des personnages romanesques de Bauchau. De son long séjour en Suisse a donc émergé ce nouveau rapport à soi et à son identité, qui s'avère non seulement déterminant dans les œuvres écrites à cette époque mais qui l'est également dans celles qui lui seront postérieures - pensons notamment à CEdipe, qui, à la fin du récit, "est encore, est toujours sur la route» ${ }^{\mathbf{9 6}}$.

Isabelle Vanquaethem

FNRS/Université catholique de Louvain

\footnotetext{
93 Jean-Claude Bonnet, "Le fantasme de l'écrivain", dans Poétique, n63, 1985, pp. 259-277.

94 Paul Bénichou, Le Sacre de l'écrivain, 1750-1830. Essai sur l'avènement d'un pouvoir spirituel laïc dans la France moderne, Paris, Corti, 1973.

95 Nathalie Heinich, op. cit., p. 312.

96 Henry Bauchau, EEdipe sur la route, Arles/Bruxelles, Actes Sud/Labor, 1992, Coll. «Babel» (rééd.), p. 380 .
} 